\title{
Actigraphy-based evaluation of sleep quality and physical activity in individuals with spinal cord injury
}

\author{
Sergiu Albu ${ }^{1} \cdot$ Guilherme Umemura $^{2}$ - Arturo Forner-Cordero ${ }^{2}$
}

Received: 28 October 2018 / Revised: 29 December 2018 / Accepted: 31 December 2018

(c) International Spinal Cord Society 2019

\begin{abstract}
Study design Cross-sectional study.

Background Sleep disturbances are frequently reported by individuals with spinal cord injury (SCI) and are associated both with poor quality of life and reduced ability to participate in rehabilitation and daily life activities.

Objectives This study investigated sleep quality based on self-reports and actigraphy in individuals with SCI as compared to able-bodied. We also explored the relationship between sleep quality, physical activity, and neuropathic pain.

Setting Institute Guttmann, Neurorehabilitation Hospital, Badalona, Barcelona, Spain.

Methods Fourteen SCI patients (12 males, $43.10 \pm 10.59$ y.o.) and 10 healthy individuals ( 7 males, mean age $46.21 \pm$ 12.58 y.o.) were enrolled in the study. Participants wore wrist actigraphs for 7 consecutive days to characterize their sleep-wake cycle, rest-activity circadian rhythm and physical activity. Sleep quality, chronotype, daytime sleepiness, neuropathic pain severity and interference were assessed based on questionnaires.

Results SCI individuals reported poorer sleep quality compared to healthy individuals. Actigraphy-based sleep measurements revealed that patients woke up later, spent more time in bed and slept longer compared to the healthy controls but did not differ significantly in the estimated sleep efficacy and number of awakenings from the able-bodied controls. In individuals with SCI greater physical activity predicted higher sleep efficacy and less awakening episodes as well as shorter sleep latency and lower sleep disturbance.

Conclusions The actigraphy-based sleep estimates indicate that patients with SCI spent more time in bed and slept longer but their sleep efficacy was similar to able-bodied controls. Maintaining regular physical activity could improve pain control and sleep quality.
\end{abstract}

\section{Introduction}

Sleep is an essential physiological need that has a critical role for general health. Sleep disturbances are more frequently reported among individuals with spinal cord injury

Supplementary information The online version of this article (https:// doi.org/10.1038/s41394-019-0149-0) contains supplementary material, which is available to authorized users.

Sergiu Albu

sergiualbumed@yahoo.com

1 Institute Guttmann, Neurorehabilitation Hospital, Badalona 08916 Barcelona, Spain

2 Biomechatronics Laboratory, Mechatronics and Mechanical Systems Department, Escola Politécnica, University of São Paulo, São Paulo, Brazil
(SCI) compared to healthy individuals, and are associated with negative health outcomes, poor quality of life [1-4].

Most studies in SCI patients have focused on sleeprelated breathing disorders that are critical in individuals with tetraplegia, although alterations in circadian rhythm have also been described [5]. Self-reported measures and sleep diaries are widely used to identify general sleep patterns and sleep efficiency. However, they are not reliable instruments because they are subject to recall biases [6]. Laboratory-based polysomnography (PSG) has long been considered the gold standard to measure sleep objectively, but is unfeasible for long-term and home utilization [7]. Actigraphic monitors, which incorporate a small accelerometer that detects body movements, are an alternative to PSG to estimate sleep-wake cycle. Some available actigraphs also record different type of light sources and body temperature, contributing to a better estimation of sleep and circadian rhythms. In addition, they allow for long-term 
sleep monitoring over several weeks or months [8]. Validation studies of actigraphic monitoring over 3 weeks in clinical and nonclinical populations concluded that actigraphy is a useful and valid means for estimating total sleep time and wakefulness after sleep onset with an overall participant-specific accuracy above 80\% [9]. There are evidence-based recommendations for the use of actigraphy in the clinical setting to estimate total sleep time, to characterize circadian rhythms and sleep patterns, and to assess response to therapy [10]. Although the severity of spinal lesion can affect the general mobility of SCI patients, normal arm movements in patients with injury below the C5-C7 level support the use of wrist actigraphy for sleep-wakefulness assessment in this population [2].

Among SCI individuals, the main factors reported to interfere with sleep quality include spasms, pain, paraesthesia, bowel, and bladder routines, limited ability to position themselves comfortably for sleep, either due to paralysis or pressure ulcer prevention and daytime sleepiness due to pain or antispasticity medication $[1,11]$. In addition, sleep disturbances might be exacerbated by the hospital environment [2], interfering with patients' ability to participate in rehabilitation and daily life activities [11, 12]. Thus, the current study evaluated sleep quality based on self-reports and actigraphy in hospitalized individuals with paraplegia secondary to SCI as compared to healthy controls. We hypothesized that patients would present difficulties falling asleep and/or staying asleep, would more often wake up during the night, have shorter sleep duration, and report more daytime sleepiness as compared to ablebodied. Wheelchair users with a SCI, who underwent a locomotor training program, perceived improvements in their sleep quality [13], whereas regular physical activity contributed to a better pain control, improvements in their overall health status and life satisfaction in this population [14-17]. Actigraphy is considered a suitable method of measuring physical activity in people with SCI and has concurrent validity with a self-report measure of activity intensity and frequency in wheelchair users [18]. Therefore, we also explored the relationship between sleep quality, physical activity and neuropathic pain (NP). We hypothesized that persons with more intense physical activity would show less severe NP and better sleep quality.

\section{Methods}

\section{Participants}

Patients with SCI were included if they were 18-65 years of age; had paraplegia secondary to SCI below the Th2 level and were followed an intensive rehabilitation program. The program included exercises for the whole body: locomotor training, exercises to improve trunk control, moving in bed and transfering into and out of a wheelchair, lower limb musculature strengthening, assisted cycling on a stationary bike, and upper body strength and fitness program, at least $5 \mathrm{~h}$ daily during weekdays. Individuals were excluded if they presented cervical SCI, limitation in upper limb movements due to associated musculoskeletal pain/injury, associated brain injury or severe systemic disorders (e.g., cardiovascular disease, cancer, any infection) that could limit their participation in physical exercises. The severity and the level of SCI were assessed according to the American Spinal Cord Injury Association Impairment Scale (AIS) and the International Standards for Neurological Classification of Spinal Cord Injury [19]. Fourteen patients (12 males and 2 females, mean age $43.10 \pm 10.59$ years) were included in the study.

Ten age-matched volunteers ( 7 males and 3 females, mean age $46.21 \pm 12.58$ years) without neurological conditions were enrolled as controls. Individuals reporting sleep problems or those taking medication or recreational drugs on a regular basis were not included in the study.

\section{Study design and protocol}

Participants were asked to wear a wrist Actigraph (ActTrust $^{\circledR}$, Condor Instruments Ltda, SP, Brazil) on the nondominant arm as a regular watch continuously for 7 consecutive days (except during bathing). After returning the actigraph participants were asked to fill out a series of questionnaires to characterize their sleep quality, chronotype, daytime sleepiness, and the type and severity of neuropathic pain.

\section{Actigraph}

The wrist Actigraph (ActTrust ${ }^{\circledR}$, Condor Instruments Ltda, SP, Brazil) registers motion by means of 3-axis accelerometry. In addition, it integrates environmental light and skin temperature sensors that increase the accuracy of estimation of the sleep-wake cycle. The devices were configured to register the motion activity, to process it with a Proportional Integral Mode (PIM) algorithm (1-minute epoch) and to calculate variables that measure the circadian rhythm and sleep-wake cycle regularity. The PIM integrates the acceleration signal to provide a measurement of the area under the curve and it is considered to provide the best possible estimation of sleep and wake states for actimetry devices. This device has been validated in previous studies [20, 21] on a group of patients who underwent a polysomnography study for one night for suspected sleep disorders. In the preliminary study with 43 patients, the device showed $83 \%$ sensitivity, $69 \%$ specificity and $89 \%$ accuracy of identification of sleep in agreement with polysomnography data. 


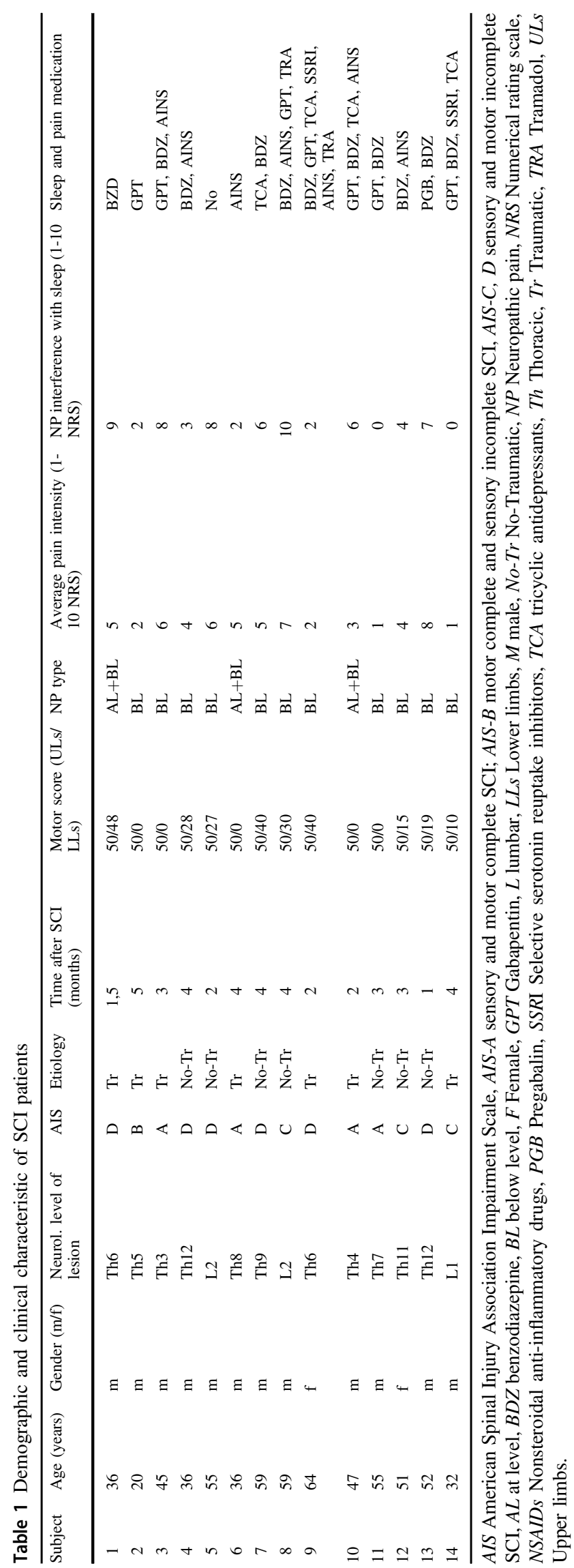

Sleep-wake cycle and physical activity analysis

Information from the Actigraph was downloaded to a computer using an Actigraph reader (Condor Instruments, SãoPaulo, Brazil). The data were analyzed using the software ActStudio (Condor Instruments Ltda., SP, Brazil) which allows for extracting, visualizing and exporting of collected data, based on the Cole-Kripke algorithm.

The following sleep parameters were measured: bedtime (time when the person was trying to sleep, related to absence of light sources and reduced movements), latency to sleep onset (the period from the bed time until the beginning of sustained movements of subthreshold amplitude at night), wake up time (sustained movements of suprathreshold amplitude in the morning), time in bed (time between bedtime and final wake up), sleep time (time between sleep onset and final wake up excluding periods of nighttime awakening), wake after sleep onset (WASO, represents the duration of nighttime awakenings), and sleep efficacy (the total sleep time divided by the time in bed, expressed as a percentage). Multiple components were measure to characterize the rest-activity circadian rhythm. Intradaily variability (IV and IV60), was monitored to quantify the rhythm fragmentation to the 24-h light-dark cycle and $60 \mathrm{~min}$ periods respectively (higher fragmentations suggests poor sleep, daytime naps or nocturnal activity) and Interdaily stability (IS and IS60), was monitored to quantify the synchronization to the 24-h light-dark cycle and $60 \mathrm{~min}$ periods respectively (higher values indicate better sleep quality, less fragmentation or nocturnal activity) [22]. Activity was measured by 3 variables: M10 (the most active $10 \mathrm{~h}$ period), L5 (the average amplitudes of the least active $5 \mathrm{~h}$ period) and the $\mathrm{Ra}$ (relative amplitude calculated as (M10 - L5)/(M10 + L5), with higher values indicating a greater daytime activity and reduced activity during sleep).

\section{Self-report sleep evaluation}

The Pittsburgh Sleep Quality Index [23], the Epworth sleepiness scale [24], and the Morningness Eveningness Questionnaire [25] were employed to evaluate different aspects of sleep quality, daytime sleepiness and chronotype. (A more detailed description of the questionnaires can be found in the supplementary information).

\section{Neuropathic pain}

NP was evaluated according to the recommendations for the diagnosis and the grading system of the NP for clinical and research purposes [26]. The NP was classified as at-level or below-level according to the International Spinal Cord Injury Pain criteria [27]. The type and severity of the NP 
was evaluated using the Neuropathic Pain Symptoms Inventory [28] whereas the variability in pain intensity and the interference with different daily life activities were evaluated employing the Brief Pain Inventory [29]. (A more detailed description of the questionnaires can be found in the supplementary information).

\section{Medication}

The use of sleep medication, analgesics, antiepileptics, and antidepressants was recorded (BDZ: benzodiazepine; GPT: Gabapentin; PGB; Pregabalin; TCA; tricyclic antidepressants; SSRI: Selective serotonin reuptake inhibitors; NSAID: Nonsteroidal anti-inflammatory; TRA: Tramadol).

\section{Statistical analysis}

Statistical analysis was performed with a commercial software package (IBM SPSS, version 23, SPSS Inc., Chicago, IL, USA). The Shapiro-Wilk's test was used to examine normality of the data. The independent-samples t-test was used to evaluate between-groups differences in demographic and sleep variables. The PSQI sub-scores violated the assumption of normal distribution; therefore, the Mann-Whitney $U$-test was employed when comparing variables between groups. A Bonferroni correction was used for multiple comparisons. Linear regression analyses were conducted to evaluate whether the intensity of specific type of neuropathic pain and physical activity indices predicted sleep parameters. Data are presented as mean and standard deviation (SD). A two-tailed test with an alpha level of .05 was used for all analyses.

\section{Results}

\section{Participants' characteristics}

Demographic and clinical characteristics of the SCI participants are presented in the Table 1. Participants included 4 individuals with complete and 10 persons with incomplete SCIs below the Th3 spinal segment, and were enrolled in the study an average of $3.04 \pm 0.18$ months after SCI. Twelve patients reported at level and/or below level neuropathic pain and 2 patients reported dysesthesia/paresthesia below the level of spinal lesion. Eleven patients were taking benzodiazepines for sleep alterations.

\section{Questionnaire-based sleep-wake cycle}

Questionnaires-based sleep parameters are presented in the Table 2.
Table 2 Questionnaire-based sleep parameters

\begin{tabular}{|c|c|c|c|c|c|}
\hline & \multicolumn{2}{|c|}{ Healthy } & \multicolumn{2}{|l|}{ SCI } & \multirow[t]{2}{*}{$P$-value } \\
\hline & Mean & SD & Mean & SD & \\
\hline Bedtime & 23:09 & $0: 47$ & $21: 49$ & $1: 29$ & 0.01* \\
\hline $\begin{array}{l}\text { Latency to sleep } \\
\text { onset (min) }\end{array}$ & $00: 24$ & $0: 12$ & 01:07 & $1: 10$ & $\mathbf{0 . 0 3} *$ \\
\hline Wake up time & $6: 57$ & $0: 47$ & $7: 51$ & $1: 02$ & $0.025 *$ \\
\hline $\begin{array}{l}\text { Time in bed (h: } \\
\text { min) }\end{array}$ & $7: 49$ & $0: 53$ & $9: 57$ & $1: 50$ & 0.001* \\
\hline $\begin{array}{l}\text { Sleep duration (h: } \\
\text { min) }\end{array}$ & $6: 39$ & $0: 42$ & $6: 30$ & $1: 24$ & 0.73 \\
\hline Sleep Quality & 1.20 & 0.42 & 1.71 & 0.83 & 0.96 \\
\hline Sleep Latency & 1.20 & 0.63 & 2.07 & 0.92 & $\mathbf{0 . 0 1 9}^{\#}$ \\
\hline Sleep Duration & 1.20 & 0.63 & 1.36 & 1.22 & 0.80 \\
\hline Sleep Efficacy & 0.60 & 0.70 & 1.93 & 1.27 & $\mathbf{0 . 0 1 6}^{\#}$ \\
\hline Sleep Disturbance & 1.50 & 0.53 & 1.57 & 0.51 & 0.80 \\
\hline Sleep Drug Use & 0.00 & 0.00 & 1.86 & 1.46 & $0.007^{\#}$ \\
\hline $\begin{array}{l}\text { Daytime } \\
\text { Disfunction }\end{array}$ & 0.80 & 0.63 & 1.07 & 0.83 & 0.51 \\
\hline PSQI total score & 6.50 & 2.01 & 11.57 & 4.62 & $0.007 *$ \\
\hline ESS Score & 5.90 & 2.42 & 7.57 & 5.95 & 0.36 \\
\hline MEQ Score & 57.50 & 7.68 & 55.14 & 8.56 & 0.49 \\
\hline
\end{tabular}

*Independent-samples $t$-test

"Mann-Whitney $U$-test

SCI individuals reported going to bed earlier than controls $(p=0.018)$ and waking up later $(p=0.025)$ and therefore spent more time in bed $(p=0.025)$. However, their perception of sleep duration was similar to the healthy group $(p=0.73)$. As indicated by PSQI total score the SCI group had poorer self-reported sleep quality compared to healthy individuals $(p=0.007)$. This was determined by longer latency to sleep onset $(p=0.019)$, reduced sleep efficacy $(p=0.016)$ and the use of sleep medication $(p=$ 0.007).

\section{Actigraphy-based sleep-wake cycle}

Actigraphy-based sleep parameters (see Table 3) indicated that both healthy and SCI individuals went to bed at a similar time $(p=0.17)$. However, SCI patients woke up later compared to healthy controls $(p=0.006)$ and therefore spent more time in bed $(p=0.001)$. Although patients slept longer $(p=0.02)$ their sleep efficacy was similar to the control group $(p=0.40)$. Furthermore, there were no differences in the number of awakenings $(p=0.93)$.

Comparison between questionnaire and actigraphy-based sleep measures indicated significant differences between wake up time $(p=0.002)$ and sleep duration $(p=0.001)$. Furthermore, we found no significant correlations between 
Table 3 Actigraphy-based sleep parameters

\begin{tabular}{|c|c|c|c|c|c|}
\hline & \multicolumn{2}{|l|}{ Healthy } & \multicolumn{2}{|l|}{ SCI } & \multirow[t]{2}{*}{$P$-value } \\
\hline & Mean & SD & Mean & SD & \\
\hline Bedtime & $22: 58$ & $0: 59$ & $22: 22$ & 1:06 & 0.17 \\
\hline $\begin{array}{l}\text { Sleep latency (h: } \\
\text { min) }\end{array}$ & $0: 04$ & 0:05 & 0:06 & $0: 12$ & 0.60 \\
\hline Wake up time & $7: 51$ & $0: 40$ & $8: 42$ & $0: 37$ & 0.006* \\
\hline $\begin{array}{l}\text { Time in bed (h: } \\
\text { min) }\end{array}$ & $8: 52$ & $0: 40$ & $10: 12$ & 1:04 & 0.001* \\
\hline $\begin{array}{l}\text { Sleep duration (h: } \\
\text { min) }\end{array}$ & $7: 20$ & $0: 31$ & $8: 17$ & $1: 14$ & $0.02 *$ \\
\hline Sleep Efficacy (\%) & 83.68 & 4.47 & 81.63 & 7.29 & 0.40 \\
\hline WASO & $1: 15$ & $0: 33$ & $1: 41$ & $0: 38$ & 0.09 \\
\hline Awake & 11.84 & 5.01 & 11.66 & 4.77 & 0.93 \\
\hline Mean M10 & 4329.55 & 1236.44 & 3891.17 & 1283.65 & 0.41 \\
\hline Onset M10 & 9:05 & 1:06 & $8: 53$ & $0: 42$ & 0.62 \\
\hline Mean L5 & 396.84 & 216.87 & 291.899 & 224.07 & 0.26 \\
\hline Onset L5 & 3:09 & $1: 12$ & $2: 33$ & $1: 34$ & 0.30 \\
\hline Mean RA & 0.84 & 0.11 & 0.88 & 0.07 & 0.30 \\
\hline IV & 0.75 & 0.16 & 0.67 & 0.08 & 0.11 \\
\hline IV60 & 0.91 & 0.31 & 0.66 & 0.11 & $\mathbf{0 . 0 3} *$ \\
\hline IS & 0.39 & 0.11 & 0.51 & 0.09 & $0.009 *$ \\
\hline IS60 & 0.47 & 0.14 & 0.63 & 0.11 & 0.006* \\
\hline
\end{tabular}

*Independent-samples $t$-test

similar questionnaires based and actigraphy-based sleep variables in the SCI or healthy group.

In the control group actigraphy-based measurements indicated that later bedtime predicted higher sleep efficacy $(\mathrm{F}(1,8)=5.97, p=0.04, \mathrm{R} 2=0.42)$ whereas in the SCI group later bedtime predicted less awakening episodes $(\mathrm{F}(1$, $12)=6.29, p=0.028, \mathrm{R} 2=0.34)$.

Examples of actigraphic monitoring in a healthy individual (Fig.1) and a SCI patient (Fig. 2).

\section{Actigraph-based circadian rest-activity rhythm}

Compared to healthy controls SCI individuals had less fragmented (IV60) $(p=0.033)$ and more synchronized circadian rhythm (IS, IS60) $(p=0.009$ and $p=0.006$ respectively) (see Table 3). Regression analysis revealed that in the SCI group, more fragmented circadian rhythm (higher IV60) predicted higher PSQI score $(\mathrm{F}(1,12)=4.85$, $p=0.048, \mathrm{R} 2=0.28$ ). In addition, more synchronized sleep (higher IS) predicted morningness chronotype $\mathrm{F}(1$, 12) $=9.70, p=0.009, \mathrm{R} 2=0.45)$.

\section{Chronotype and daytime sleepiness}

Based on MEQ score, most participants were intermediate type (9 out of 14 SCI individuals and 6 out of 10 healthy individuals). Excessive daytime sleepiness (ESS score $\geq 10$ ) was found in 4 SCI individuals and 2 healthy individuals. However, there were no statistically significant differences between groups in chronotype $(p=0.49)$ or daytime sleepiness $(p=0.36)$.

\section{Physical activity and sleep quality}

All patients were involved in an intensive rehabilitation program which included at least $5 \mathrm{~h} /$ day of physical activity. Although the amplitude of the most active and least active periods, as measured by actigraphy (M10, L5), were lower in individuals with SCI compared to healthy controls, these differences were not statistically significant.

We found no significant correlations between ASIA motor score and activity measures (M10, L5, and RA) in the SCI group. Regression analysis revealed that more intense physical activity (higher M10 and L5) predicted shorter sleep latency sub score $[(\mathrm{F}(1,12)=5.40, p=0.039, \mathrm{R} 2=$ $0.31)$ and $(\mathrm{F}(1,12)=5.42, p=0.038, \mathrm{R} 2=0.31)$, respectively] as well as shorter sleep duration $[(\mathrm{F}(1,12)=14.03$, $p=0.003, \mathrm{R} 2=0.54)$ and $(\mathrm{F}(1,12)=14.55, p=0.002$, $\mathrm{R} 2=0.55)$ ]. In addition, greater RA (indicating a greater daytime activity and reduced activity during sleep) predicted lower sleep disturbance subscore on the PSQI ( $\mathrm{F}(1$, $12)=5.40, p=0.039, \mathrm{R} 2=0.31)$, higher sleep efficacy $(\mathrm{F}$ $(1,12)=19.91, p=0.01, \mathrm{R} 2=0.62)$ and less awakening episodes $(\mathrm{F}(1,12)=7.39, p=0.019, \mathrm{R} 2=0.38)$.

\section{Pain and sleep quality}

Twelve patients presented either below level or at level neuropathic pain and 2 individuals presented paresthesia/ dysesthesia below the level of injury. More intense nonevoked continuous pain predicted lower interdaily stability $[\mathrm{IS}(\mathrm{F}(1,12)=9.72, p=0.009, \mathrm{R} 2=0.45), \operatorname{IS60}(\mathrm{F}(1,12)$ $=11.75, p=0.005, \mathrm{R} 2=0.50)]$ and shorter perceived sleep duration $(\mathrm{F}(1,12)=15.30, p=0.002, \mathrm{R} 2=0.56)$.

\section{Discussion}

The current study characterized self-reports and actigraphy-based sleep alterations in individuals with SCI and explored whether physical activity and neuropathic pain interfere in sleep quality. The actigraphy-based sleep estimations indicate that patients spent more time in bed and slept longer compared to healthy controls whereas sleep efficacy was similar between groups. These measures were inconsistent with self-reported sleep parameters. In addition, we found that lower physical activity and more intense continuous neuropathic pain were related with poor sleep quality. 


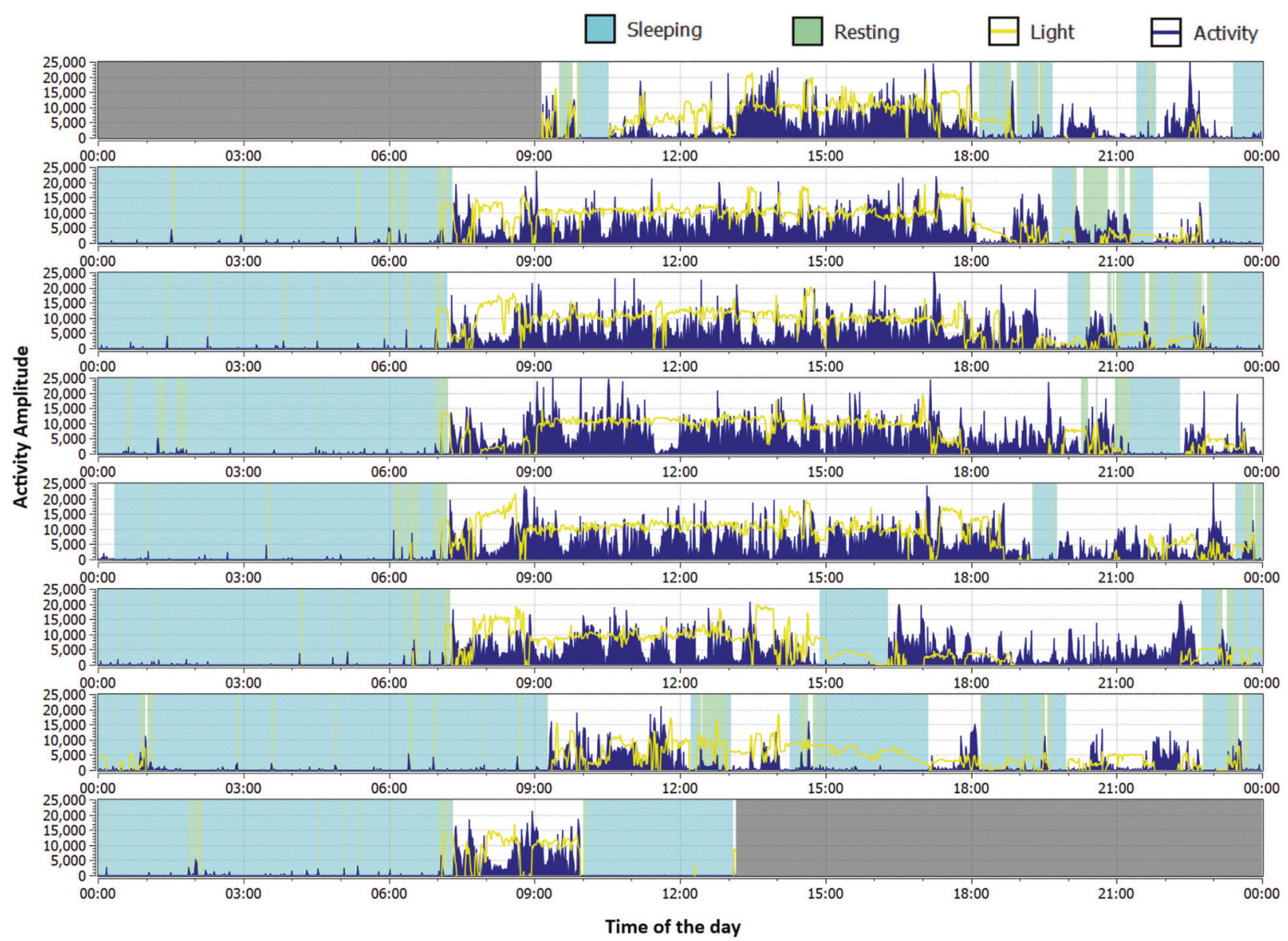

Fig. 1 Healthy individual, male, 51 years. Pittsburgh score = 8. MEQ $=56, \mathrm{ESS}=5$. Questionnaire-based sleep parameters: Bedtime-23:30; Sleep latency-20 min; Wake up time-7:00; Time in bed- 7:30 h; Sleep duration-6:00 h; Sleep efficacy-80.00\%. Actigraphy-based sleep parameters: Bedtime-21:34; Sleep latency-1 min; Wake up time-7:27; Time in bed- 9:53 h; Sleep duration-8:02 h; Sleep efficacy-82.55\%; Awakenings-11.86; Mean M10-6133.74; MeanL5-371.04; Mean RA0.90; IV-0.52; IV60-0.54; IS-0.57; IS60-0.67

\section{Sleep alterations in SCI}

As indicated by the PSQI global score, SCI patients reported poorer sleep compared to the healthy controls. This was characterized by longer time in bed, longer sleep latency, and lower sleep efficacy, which is in line with previous research $[1,2,11,16]$. Further, the actigraphybased sleep measurements in our study revealed that patients spent more time in bed and slept longer whereas their sleep latency and sleep efficacy were similar to the healthy controls. Compared to polysomnography, actigraphy tends to underestimate sleep-onset latency while sleep diaries overestimated it [30]. Similar discordance between self-reports and actigraphy-based measures of sleep were previously described in individuals with SCI who referred worsening of sleep quality although their score on mini sleep questionnaire and actigraphy-based measures of sleep (except for the number of awakenings) were comparable to the healthy subjects [2]. Whereas selfreported and actigraphy-based estimation of time in bed were similar in our study, the actigraphy-based estimated sleep duration was significantly greater compared to selfreported sleep duration. When using actigraphy, identification of specific sleep periods is based on identification of arm movements of subthreshold/suprathreshold amplitude. Thus, sleep duration might be misestimated because some insomniac patients, for example, often complain of staying awake but remain immobile in bed [31]. In addition, our results revealed a non-significant decrease of the mean amplitude of movements during the most active and less active periods (M10 and L5) in the SCI group, which might have contributed to the overestimation of the time in bed and sleep duration [32]. However, time estimation is a complex cognitive task, which is especially challenging when the period includes sleep [33]. This complexity can lead to erroneous estimation of sleep latency, duration and the number of awakenings in comparison to laboratory sleep measures [34]. Subjective underestimation of sleep duration could also explain the lower sleep efficacy subscore on the PSQI in SCI patients since the latter is 


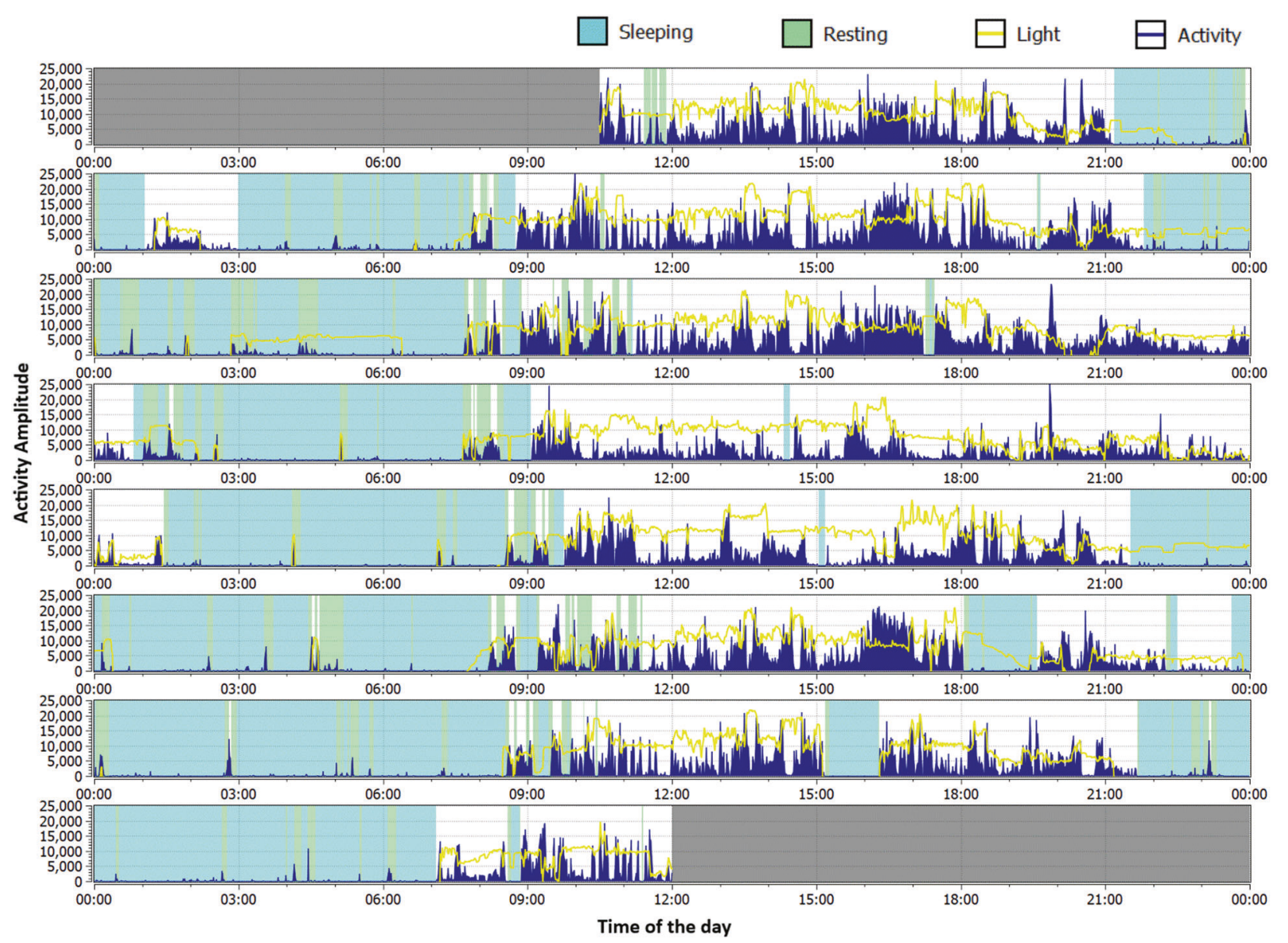

Fig. 2 SCI individual, male, 55 years, 2 months after no-traumatic SCI, ASIA-D, neurologic level of injury L2, 50/27 motor score (upper/ lower limbs), disesthesia-paresthesia below the level of spinal injury of an average intensity $6(0-10 \mathrm{NRS})$ and sleep interference $8(0-10$ NRS). No analgesics or sleep medication. Pittsburgh score $=12$. $\mathrm{MEQ}=52$. $\mathrm{ESS}=7$. Questionnaire-based sleep parameters: Bedtime-

calculated as "percentage of total sleep time out of the total time spent in bed" whereas other indicators of good sleep such as sleep quality, sleep disturbance and daytime dysfunction sub scores were not different compared to healthy controls. Excessive daytime sleepiness is an indicator of insufficient sleep or poor sleep efficiency [35]. In addition, most patients in our study were taking antispastic, antiepileptic, opioids or benzodiazepines, which are common causes of daytime sleepiness and can alter person's participation in daily life and rehabilitation activities. However, we found no significant differences in the Epworth score and chronotype between groups. Furthermore, compared to healthy controls, the SCI individuals had less fragmented and more synchronized circadian rhythm, which are indicative of better sleep quality [22]. However, we must consider that adherence to the hospital schedule during an intensive rehabilitation program might play a significant role in circadian rhythm synchronization, which has been
22:00; Sleep latency-10 min; Wake up time-7:30; Time in bed-9:30 h; Sleep duration-6:00 h; Sleep efficacy-63.16\%. Actigraphy-based sleep parameters: Bedtime-23:22; Sleep latency-1 min; Wake up time-8:35; Time in bed- 9:12 h; Sleep duration-6:51 h; Sleep efficacy-76.51\%; Awakenings-9.75; Mean M10-4658.37; MeanL5-497.13; Mean RA0.84; IV-0.63; IV60-0.63; IS-0.60; IS60-0.75

shown to affect motor performance in healthy individuals [36].

\section{Physical activity, pain, and sleep quality}

A recent meta-analysis revealed that physical activity improves PSQI score and the Insomnia Severity Index [37]. Actigraphs can also be used to evaluate physical activity. Research evidence suggests a strong relationship between wrist acceleration and physical activity and energy expenditure in free-living adults [38]. Accelerometer device worn on the upper arm or on the wrist can also assist manual wheelchair users, including individuals with SCI, to estimate physical activity and energy expenditure [39, 40]. Based on wrist actigraphy measures, we found no significant differences in parameters of physical activity (M10 and L5) between SCI and healthy individuals, supporting the use of actigraphy in individuals with SCI below Th2. 
Participation in an exercise training program has showm positive effects on sleep quality, reduced sleep latency and medication use in adults with sleep problems [41]. The linear regression analyses in our study revealed that higher intensity of physical activity in SCI individuals predicted higher sleep effectiveness and less awakening episodes as well as shorter latency and lower sleep disturbance (PSQI) which suggest that participation in an intensive rehabilitation program could potentially improve sleep quality. In addition, higher intensity of continuous neuropathic pain in our population was associated with increased sleep fragmentation. In outpatients with SCI, continuous pain remains to be one of the main predictors for poor sleep quality [12, 16]. Short-term and long-term physical activities in wheelchair-dependent individuals with SCI decreased the intensity of neuropathic pain and improved negative mood $[16,17]$. Therefore, regular exercises should be encouraged in SCI patients to improve sleep quality and pain control.

\section{Limitations}

Limitations of the current study should be addressed. Although the actigraphy allows estimation of wake-sleep cycle, this method does not evaluate sleep stages and therefore does not substitute PSG in patients with specific sleep alterations. Actigraphy-based data were partially inconsistent with self-reported sleep parameters, which could be related to the non-significant reduction of physical activity parameters (M10 and L5) in SCI individuals or erroneous subjective time estimation. Drug-related sleep changes should also be considered as most participants in the SCI group were taking benzodiazepines whereas none of the individuals in the control group was taking sleep medication. Another limitation is the estimation of physical activity based on actigraphy in absence of specific energy expenditure quantification. In addition, the small sample size and the prevalence of male individuals limit the generalizability of our findings.

\section{Conclusions}

Our main results revealed that according to actigraphybased sleep estimates patients spent more time in bed and slept longer but did not differ significantly in the estimated sleep efficacy and number of awakenings from the ablebodied controls. The strong relationship between the reduced physical activity and more intense continuous neuropathic pain and poor sleep quality supports the inclusion of SCI patients in regular physical activities that could potentially improve pain control and sleep quality.

\section{Data archiving}

Data available on request. Requests should be submitted to the Research Committee of Institute Guttmann.

Funding The authors received no specific funding for this work.

Author contributions SA and AFC were responsible for conceptual framing of the study. SA collected the data. SA and GU conducted data analyses and all authors discussed the results. SA and GU drafted the manuscript and all authors revised it critically for important intellectual content. All authors approved the final version.

\section{Compliance with ethical standards}

Conflict of interest The authors declare that they have no conflict of interest.

Statement of ethics This study was approved by the Clinical Research Ethics Committee of the Institute Guttmann and has been conducted in accordance with the Declaration of Helsinki for ethical conduct and report of research.

Publisher's note: Springer Nature remains neutral with regard to jurisdictional claims in published maps and institutional affiliations.

\section{References}

1. Biering-Sorensen F, Biering-Sorensen M. Sleep disturbances in the spinal cord injured: an epidemiological questionnaire investigation, including a normal population. Spinal Cord. 2001;39:505-13.

2. Spivak E, Oksenberg A, Catz A. The feasibility of sleep assessment by actigraph in patients with tetraplegia. Spinal Cord. 2007;45:765-70.

3. Spong J, Graco M, Brown DJ, Schembri R, Berlowitz DJ. Subjective sleep disturbances and quality of life in chronic tetraplegia. Spinal Cord. 2015;53:636-40.

4. Widerstrom-Noga EG, Felipe-Cuervo E, Yezierski RP. Chronic pain after spinal injury: interference with sleep and daily activities. Arch Phys Med Rehabil. 2001;82:1571-7.

5. Giannoccaro MP, Moghadam KK, Pizza F, Boriani S, Maraldi NM, Avoni P, et al. Sleep disorders in patients with spinal cord injury. Sleep Med Rev. 2013;17:399-409.

6. Fichten CS, Creti L, Amsel R, Bailes S, Libman E. Time estimation in good and poor sleepers. J Behav Med. 2005;28:537-53.

7. Van de Water AT, Holmes A, Hurley DA. Objective measurements of sleep for non-laboratory settings as alternatives to polysomnography--a systematic review. J Sleep Res. 2011;20:183-200.

8. Bellone GJ, Plano SA, Cardinali DP, Chada DP, Vigo DE, Golombek DA. Comparative analysis of actigraphy performance in healthy young subjects. Sleep Sci. 2016;9:272-9.

9. Marino M, Li Y, Rueschman MN, Winkelman JW, Ellenbogen JM, Solet JM, et al. Measuring sleep: accuracy, sensitivity, and specificity of wrist actigraphy compared to polysomnography. Sleep. 2013;36:1747-55.

10. Morgenthaler T, Alessi C, Friedman L, Owens J, Kapur V, Boehlecke B, et al. Practice parameters for the use of actigraphy in the assessment of sleep and sleep disorders: an update for 2007. Sleep. 2007;30:519-29. 
11. Fogelberg DJ, Leland NE, Blanchard J, Rich TJ, Clark FA. Qualitative experience of sleep in individuals with spinal cord injury. OTJR (Thorofare N J). 2017;37:89-97.

12. January AM, Zebracki K, Chlan KM, Vogel LC. Poor sleep in adults with pediatric-onset spinal cord injury: associations with pain, health, and activity. J Spinal Cord Med. 2017;40:560-6.

13. Gagnon DH, Vermette M, Duclos C, Aubertin-Leheudre M, Ahmed S, Kairy D. Satisfaction and perceptions of long-term manual wheelchair users with a spinal cord injury upon completion of a locomotor training program with an overground robotic exoskeleton. Disabil Rehabil Assist Technol. 2017;19:1-8.

14. Hetz SP, Latimer AE, Buchholz AC, Martin Ginis KA, Group SSR. Increased participation in activities of daily living is associated with lower cholesterol levels in people with spinal cord injury. Arch Phys Med Rehabil. 2009;90:1755-9.

15. Buchholz AC, Martin Ginis KA, Bray SR, Craven BC, Hicks AL, Hayes $\mathrm{KC}$, et al. Greater daily leisure time physical activity is associated with lower chronic disease risk in adults with spinal cord injury. Appl Physiol Nutr Metab. 2009;34:640-7.

16. Norrbrink Budh C, Hultling C, Lundeberg T. Quality of sleep in individuals with spinal cord injury: a comparison between patients with and without pain. Spinal Cord. 2005;43:85-95.

17. Sato G, Osumi M, Morioka S. Effects of wheelchair propulsion on neuropathic pain and resting electroencephalography after spinal cord injury. J Rehabil Med. 2017;49:136-43.

18. Warms CA, Belza BL. Actigraphy as a measure of physical activity for wheelchair users with spinal cord injury. Nurs Res. 2004;53:136-43.

19. Kirshblum SC, Burns SP, Biering-Sorensen F, Donovan W, Graves DE, Jha A, et al. International standards for neurological classification of spinal cord injury (revised 2011). J Spinal Cord Med. 2011;34:535-46.

20. Rodrigues JL, Volpe AL, Padilha CL, Eckeli AL Actigraphy validation: preliminar analysis. Meeting of the Associated Professional Sleep Societies; 2016; Denver: Sleep; 2016. p. a372.

21. Rodrigues JL Validação de um actigrafo nacional.: University of São Paulo; 2017.

22. Goncalves BS, Cavalcanti PR, Tavares GR, Campos TF, Araujo JF. Nonparametric methods in actigraphy: an update. Sleep Sci. 2014;7:158-64.

23. Buysse DJ, Reynolds CF 3rd, Monk TH, Berman SR, Kupfer DJ. The Pittsburgh Sleep Quality Index: a new instrument for psychiatric practice and research. Psychiatry Res. 1989;28:193-213.

24. Johns MW. A new method for measuring daytime sleepiness: the Epworth sleepiness scale. Sleep. 1991;14:540-5.

25. Horne JA, Ostberg O. A self-assessment questionnaire to determine morningness-eveningness in human circadian rhythms. Int $\mathbf{J}$ Chronobiol. 1976;4:97-110.
26. Treede RD, Jensen TS, Campbell JN, Cruccu G, Dostrovsky JO, Griffin JW, et al. Neuropathic pain: redefinition and a grading system for clinical and research purposes. Neurology. 2008;70:1630-5.

27. Bryce TN, Biering-Sorensen F, Finnerup NB, Cardenas DD, Defrin R, Lundeberg $\mathrm{T}$, et al. International spinal cord injury pain classification: part I. Background and description. March 6-7, 2009. Spinal Cord. 2012;50:413-7.

28. Bouhassira D, Attal N, Fermanian J, Alchaar H, Gautron M, Masquelier E, et al. Development and validation of the Neuropathic Pain Symptom Inventory. Pain. 2004;108:248-57.

29. Cleeland CS, Ryan KM. Pain assessment: global use of the Brief Pain Inventory. Ann Acad Med Singap. 1994;23:129-38.

30. Vallieres A, Morin CM. Actigraphy in the assessment of insomnia. Sleep. 2003;26:902-6.

31. Natale V, Plazzi G, Martoni M. Actigraphy in the assessment of insomnia: a quantitative approach. Sleep. 2009;32:767-71.

32. Forner-Cordero A, Umemura GS, Furtado F, Gonçalves BSB. Comparison of sleep quality assessed by actigraphy and questionnaires to healthy subjects. Sleep Sci. 2018;11:141-45.

33. Bianchi MT, Wang W, Klerman EB. Sleep misperception in healthy adults: implications for insomnia diagnosis. J Clin Sleep Med. 2012;8:547-54.

34. Carskadon MA, Dement WC, Mitler MM, Guilleminault C, Zarcone VP, Spiegel R. Self-reports versus sleep laboratory findings in 122 drug-free subjects with complaints of chronic insomnia. Am J Psychiatry. 1976;133:1382-8.

35. Slater G, Steier J. Excessive daytime sleepiness in sleep disorders. J Thorac Dis. 2012;4:608-16.

36. Umemura GS, Pinho JP, da Silva Brandao Goncalves B, Furtado F, Forner-Cordero A. Social jetlag impairs balance control. Sci Rep. 2018;8:9406.

37. Banno M, Harada Y, Taniguchi M, Tobita R, Tsujimoto H, Tsujimoto $\mathrm{Y}$, et al. Exercise can improve sleep quality: a systematic review and meta-analysis. PeerJ. 2018;6:e5172.

38. White $\mathrm{T}$, Westgate $\mathrm{K}$, Wareham NJ, Brage S. Estimation of physical activity energy expenditure during free-living from wrist accelerometry in UK adults. PLoS One. 2016;11:e0167472.

39. Hiremath SV, Intille SS, Kelleher A, Cooper RA, Ding D. Estimation of energy expenditure for wheelchair users using a physical activity monitoring system. Arch Phys Med Rehabil. 2016;97:1146-53 e1.

40. Nightingale TE, Walhin JP, Thompson D, Bilzon JL. Influence of accelerometer type and placement on physical activity energy expenditure prediction in manual wheelchair users. PLoS One. 2015; 10:e0126086.

41. Yang PY, Ho KH, Chen HC, Chien MY. Exercise training improves sleep quality in middle-aged and older adults with sleep problems: a systematic review. J Physiother. 2012;58:157-63. 